\title{
BMJ open Diagnostic value of patterns of symptoms and signs of heart failure: application of latent class analysis with concomitant variables in a cross-sectional study
}

\author{
Milton Severo, ${ }^{1,2}$ Ana Rita Gaio, ${ }^{3,4}$ Patrícia Lourenço, ${ }^{5}$ Margarida Alvelos, ${ }^{5}$ \\ Alexandra Gonçalves, ${ }^{6}$ Nuno Lunet, ${ }^{1,2}$ Paulo Bettencourt, ${ }^{5}$ Ana Azevedo ${ }^{1,2,5}$
}

To cite: Severo M, Gaio AR, Lourenço P, et al. Diagnostic value of patterns of symptoms and signs of heart failure: application of latent class analysis

with concomitant variables in a cross-sectional study. BMJ Open 2012;2:e001510. doi:10.1136/bmjopen-2012001510

- Prepublication history and additional material for this paper are available online. To view these files please visit the journal online (http://dx.doi.org/10.1136/ bmjopen-2012-001510)

Received 24 May 2012 Accepted 27 September 2012

This final article is available for use under the terms of the Creative Commons Attribution Non-Commercial 2.0 Licence; see http://bmjopen.bmj.com

For numbered affiliations see end of article

Correspondence to Dr Milton Severo, milton@ med.up.pt

\section{ABSTRACT}

Objective: The diagnosis of heart failure (HF) requires a compatible clinical syndrome and demonstration of cardiac dysfunction by imaging or functional tests. Since individual symptoms and signs are generally unreliable and have limited value for diagnosing $\mathrm{HF}$, the authors aimed to identify patterns of symptoms and signs, based on findings routinely collected in current clinical practice, and to evaluate their diagnostic value, taking into account the a priori likelihood of HF.

Design: Cross-sectional evaluation.

Participants: 1115 community participants aged $\geq 45$ years from Porto, Portugal, in 2006-2008.

Main outcomes measures: Patterns were identified by latent class analysis, using concomitant variables to predict class membership. Patterns used 11 symptoms/signs, covering dimensions of congestion and hypoperfusion. Sex, age, education, obesity, diabetes and history of myocardial infarction or HF were included as concomitants.

Results: Bayesian information criteria supported a solution with three patterns: $10.1 \%$ of participants followed a pattern with symptoms of troubled breathing and signs of congestion (pattern 1), $27.8 \%$ a pattern characterised mainly by signs of congestion (pattern 2) and $62.1 \%$ were essentially asymptomatic (pattern 3); model fit was best when including concomitant variables. The likelihood ratio of patterns 1,2 and 3 for left ventricular systolic dysfunction was $3.4,1.1$ and 0.6 , and for left ventricular diastolic dysfunction $3.5,1.4$ and 0.5 , respectively.

Conclusions: The use of concomitant variables can improve the diagnostic value of the symptoms and signs patterns and, consequently, improve the usefulness of the symptoms and signs for diagnosis and as an outcome measure. The potential for application in other settings of complex diagnoses is very high. These models were shown to be useful to standardise and quantify the probabilistic reasoning in clinical diagnosis, upon which decisions of further investigation and even treatment need to be made.

\section{ARTICLE SUMMARY}

Article focus

- To identify patterns of symptoms and signs of heart failure (HF), based on findings routinely collected in current clinical practice.

- To evaluate the diagnostic value of different patterns.

- To assess the role of concomitant variable latent class models in the diagnosis of HF.

Key messages

- In this study, in a sample of the general population, three patterns were identified: 'symptomatic HF', 'signs of congestion' and 'no symptoms and signs'.

- The patterns generated relatively small changes from pretest to post-test probability of cardiac abnormalities. However, the obtained patterns showed a good diagnostic performance for exclusion of high B-type natriuretic peptide values.

- The use of concomitant variables can improve the diagnostic value of the symptoms and signs patterns and, consequently, improve the usefulness of the symptoms and signs for diagnosis and as outcome measures.

Strengths and limitations of this study

- For the first time, to our knowledge, the obtained HF classification integrated factors that have a large impact on the prevalence of symptoms and signs suggestive of HF. The model estimates the increase or decrease in class probabilities for individuals conditional on the respective concomitant variables values, contributing to an increase in the discrimination and to a decrease in the number of false-negatives and false-positives.

- The low prevalence of advanced and severe HF cases is a limitation of this study and could have underestimated the discriminative capacity of this set of symptoms and signs. 


\section{INTRODUCTION}

Heart failure (HF) is a complex clinical syndrome resulting from a variety of structural or functional cardiac disorders. The diagnosis of HF requires a compatible clinical syndrome and demonstration of cardiac dysfunction by imaging or functional tests. ${ }^{12}$ A clinical examination is always the first step in a diagnostic approach to possible $\mathrm{HF}$ and further investigation is conditional on initial clinical judgement. However, individual symptoms (such as dyspnoea and fatigue) and signs (eg, third heart sound and evidence of congestion) are generally unreliable and have limited value for diagnosing $\mathrm{HF}^{3}{ }^{3}$ Several multidimensional criteria based on symptoms and signs have been developed over decades in an attempt to standardise the clinical assessment of $\mathrm{HF}^{5-12}$ When patients initially labelled as having HF are investigated using objective-assessment criteria, only around one-third are considered to truly have HF. ${ }^{13}{ }^{14}$ Obesity, unrecognised myocardial ischaemia or pulmonary disease commonly lead to false-positive HF diagnoses. ${ }^{13}$ Additionally, it may be difficult to distinguish pathological conditions from mere physical deconditioning associated with ageing. Moreover, the varying subjective importance attributed to symptoms justifies a systematic association between reported symptoms and female gender and psychosocial characteristics, both among the healthy and those with cardiac dysfunction. Gender, age, education and obesity are major determinants of symptoms and signs suggestive of $\mathrm{HF}^{15}$ beyond their role as risk factors for $\mathrm{HF}$, and may account for false-positive and negative classification. Furthermore, the clinical judgement is modified based on the a priori likelihood of $\mathrm{HF}^{16}$ depending mainly on a history of HF or myocardial infarction, and on strong risk factors for such conditions.

Systolic HF and HF with normal left ventricular ejection are very similar at the bedside though very different when one considers cardiac structure and function, and response to therapy. About half of patients with symptomatic HF have preserved left ventricular systolic function. ${ }^{17} \mathrm{HF}$ with preserved systolic function has long been mainly an exclusion diagnosis, but the most recent guidelines require evidence of left ventricular diastolic dysfunction. ${ }^{18}$ The prevalence of HF with normal ejection fraction increased over the last years, ${ }^{19}$ in part as a consequence of increasing acknowledgement of its importance and of improvements in the ability to recognise it. Until a recent past, most clinical and epidemiological studies considered only HF with left ventricular systolic dysfunction, and community-based studies or consecutive series of patients with any kind of HF are likely to currently yield scenarios of diagnostic reasoning and validity that contrast with past cohorts.

The aims of this study were to identify patterns of symptoms and signs of HF, based on findings routinely collected in current clinical practice, including concomitant variables to predict the pattern membership; and to evaluate the diagnostic value of different patterns.

\section{METHODS}

Study design and sample selection

Participants were selected within the first follow-up of a cohort, representative at baseline of the noninstitutionalised adult population of Porto, Portugal-the EPIPorto cohort study. In 1999-2003, cohort assembly was made by random-digit dialling, using households as the sampling frame, followed by random selection of one person aged 18 years or older in each household. Refusals were not substituted within the same household. The proportion of participation was $70 \% .{ }^{20}$ At baseline, 2485 participants were recruited. Between October 2006 and July 2008, participants aged 45 years or over were eligible to a systematic evaluation of parameters of cardiac structure and function, which included a cardiovascular clinical history and physical examination, and a bidimensional transthoracic echocardiogram. Among 2048 cohort members that would be in the eligible age range at this time, $134(6.5 \%)$ had died, $198(9.7 \%)$ refused to be re-evaluated and 580 (28.3\%) were lost to follow-up (unreachable by telephone or post), and 21 (1.0\%) had missing values in at least one of the variables used in the present analysis. Therefore, $1115(54.6 \%)$ individuals aged 45 years or over were analysed to develop the new epidemiological classification scheme for clinical HF, with mean (SD) follow-up period of 7 (2.7) years. When comparing their baseline characteristics with the 933 cohort members of the same age range who were not included in the present analysis, participants were significantly younger (mean (SD) age: 57 (10) vs 59 (13) years, $\mathrm{p}<0.001$ ), had a higher level of education (median: 7 vs 4 years, $\mathrm{p}<0.001$ ), and a lower prevalence of obesity (22.5\% vs $28.9 \%, \mathrm{p}=0.001)$, hypertension $(55.3 \%$ vs $65.2 \%, \mathrm{p}<0.001)$ and previous myocardial infarction ( $2.3 \%$ vs $3.9 \%, p=0.041)$, while there were no gender differences (men: $39.0 \%$ vs $37.4 \%, \mathrm{p}=0.456$ ).

This investigation conformed to the principles expressed in the Declaration of Helsinki. The local ethics committee approved the study and participants provided written informed consent.

\section{Data collection and variables definition}

A structured questionnaire was applied by non-physician trained interviewers to obtain data on sociodemographic characteristics and lifestyles. Clinical history and physical examination were performed by physicians experienced in the management of HF patients.

The New York Heart Association classification across each set of individuals assessed by each physician was calibrated to increase the interobserver reproducibility. ${ }^{21}$

Echocardiograms were performed by trained cardiologists using the same equipment (Philips HP SONOS 5500, Philips Healthcare, Hamburg, Germany), following a standardised protocol. All measures resulted from averaging three observations.

Obesity was defined as body mass index higher than or equal to $30 \mathrm{~kg} / \mathrm{m}^{2} .^{22}$ History of myocardial infarction was defined as self-reported medical diagnosis of 
myocardial infarction or history of coronary artery bypass graft.

An overnight fasting venous blood sample was withdrawn with plasma or serum samples and used for B-type natriuretic peptide (BNP) measurement using a commercially available immunofluorimetric assay (Triage BNP Test, BIOSITE diagnostics, San Diego, California, USA). An established equation was used for the estimation of the BNP concentration in plasma when only serum was available. ${ }^{23} \mathrm{BNP}$ values were available for 630 participants and, for analysis, they were dichotomised by the cut-off point $100 \mathrm{pg} / \mathrm{ml}$, previously established for the diagnosis of $\mathrm{HF}^{24}$

Left ventricular dilation was considered when end diastolic left ventricular diameter was larger than $58 \mathrm{~mm}$ in men and $52 \mathrm{~mm}$ in women and left ventricular systolic dysfunction was defined by an ejection fraction below $45 \%$, assessed by Simpson's method, or by visual estimate. In 29 subjects (2.6\%) it was not possible to quantify the ejection fraction due to poor acoustic window.

Left ventricular hypertrophy was defined as left ventricular mass index $>110 \mathrm{~g} / \mathrm{m}^{2}$ in women and $125 \mathrm{~g} / \mathrm{m}^{2}$ in men, and left atrium dilation was defined as left atrial volume index $>40 \mathrm{ml} / \mathrm{m}^{2} .{ }^{25}$ Valvular abnormalities were considered when moderate or severe. Diastolic dysfunction was defined according to the European Society of Cardiology guidelines for HF with normal left ventricular ejection. ${ }^{18}$

\section{Statistical analysis}

Latent class analysis

Latent class analysis (LCA) is used to uncover distinct groups of individuals from a sample (patterns), homogeneous within the group, considering that the performance of an individual in a set of items is explained by a categorical latent variable with $\mathrm{K}$ classes, commonly called 'latent classes'. Interpretation of the model is usually based on item profiles in each category, obtained from the probabilities of endorsing each item response, conditional on class membership.

In this study, the number of latent classes (patterns) was defined according to the Bayesian information criterion (BIC). Starting from one single class and increasing one class at each step, the best solution was identified when the increase in the number of classes did not lead to a decrease in BIC.

LCA used 11 symptoms and signs to define a syndrome suggestive of $\mathrm{HF}$ or important for differential diagnosis, including dyspnoea, orthopnoea, nocturnal paroxysmal dyspnoea, fatigue, self-percepted and clinically confirmed oedema, hepatojugular reflux or jugular venous distension, pulmonary rates, heart murmur, trophic signs of chronic venous insufficiency and visible varicose veins.

The items selection was based on their clinical relevance for the definition of $\mathrm{HF}$ and prevalence. ${ }^{1} 218$ Other relevant signs and symptoms such as third heart sound $(0.8 \%)$, heart rate higher than 120 beats/min
$(0.1 \%)$ and hepatomegaly $(1.5 \%)$ were not taken into account because they occurred in less than $2 \%$ of the study sample.

In LCA, factors which are known to have a large impact on the prevalence of symptoms and signs suggestive of $\mathrm{HF}$ were used as concomitant variables, namely sex, age, education, obesity $(27.4 \%)$, diabetes $(13 \%)$ and history of myocardial infarction $(4 \%)$ or $\mathrm{HF}$ $(7.1 \%)$. Other relevant concomitant variables, namely smoking, alcohol intake, hypertension and history of valvular diseases were not included because they did not show a significant effect in the patterns in this sample.

In LCA, concomitant variables are covariates considered in the process of formation of the latent classes, by the multinomial regression of latent classes on concomitant variables, to allow for different contributions of the items to define the classes for different levels of concomitants. ${ }^{26}$

All LCA models were fitted using MPlus (V.5.2; Muthen \& Muthen, Los Angeles, California, USA).

\section{Patterns' diagnostic value}

To assess the diagnostic value of the defined patterns, we estimated the likelihood ratio and predictive value of patterns of symptoms and signs, with and without concomitant variables, to predict the presence of a series of outcomes, corresponding to objective structure of functional cardiac abnormalities as assessed by echocardiography at rest. The likelihood ratio measures the ratio between the prevalence of each pattern in subjects with and without the outcome. The predictive value is the a posteriori probability of the outcome, conditional on the clinical pattern.

The diagnostic value of high $\mathrm{BNP}(\mathrm{BNP} \geq 100 \mathrm{pg} / \mathrm{ml})$ was evaluated in a subgroup of the study sample with BNP measured in blood collected at the time of clinical and echocardiographic examination $(n=630)$. In untreated subjects, a concentration of BNP under $100 \mathrm{pg} / \mathrm{ml}$ has high negative-predictive value and makes HF an unlikely diagnosis. ${ }^{1}$

\section{RESULTS}

\section{Patterns of symptoms and signs of HF}

Relying only on signs and symptoms, the increase in log likelihood values levelled off when increasing from two to three and BIC reached its optimum value at three classes, supporting preference for a three-class solution. The inclusion of concomitant variables led to an improvement (decrease) in BIC values in all tested models and the three-class model was again the best solution according to BIC (table 1).

The final model with concomitant variables had the following item profiles: class 1 had high probabilities for all 11 items (symptomatic HF pattern), class 2 had high probability for volume overload and lower probability for troubled breathing (congestion pattern) and class 3 
Table 1 Latent class analysis for heart failure symptoms and signs, with and without concomitant variables (sex, age, education, obesity, diabetes mellitus and history of myocardial infarction or heart failure), in the general population aged $\geq 45$ years, Porto, Portugal, 2006-2008

\begin{tabular}{|c|c|c|c|c|c|c|c|c|}
\hline \multirow[b]{2}{*}{$\begin{array}{l}\text { Number of } \\
\text { classes } †\end{array}$} & \multicolumn{4}{|c|}{ Symptoms and signs } & \multicolumn{4}{|c|}{$\begin{array}{l}\text { Symptoms and signs with concomitant } \\
\text { variables } \S\end{array}$} \\
\hline & $\log L$ & $\begin{array}{l}\text { Number of } \\
\text { parameters }\end{array}$ & BIC & p‡ & $\log L$ & $\begin{array}{l}\text { Number of } \\
\text { parameters }\end{array}$ & BIC & pł \\
\hline \multicolumn{9}{|l|}{ LCA } \\
\hline 1 class & -5060.161 & 14 & 10218 & & & & & \\
\hline 2 classes & -4727.150 & 29 & 9657 & $<0.001$ & -4596.351 & 35 & 9438 & $<0.001$ \\
\hline 3 classes & -4613.189 & 44 & 9535 & $<0.001$ & -4438.071 & 56 & 9269 & 0.003 \\
\hline 4 classes & -4575.405 & 59 & 9564 & 0.966 & -4382.080 & 77 & 9304 & 0.770 \\
\hline \multicolumn{9}{|c|}{$\begin{array}{l}\text { TTe italic font denotes the best models according to lowest BIC. } \\
\text { fLo-Mendell-Rubin likelihood ratio test of model fit to quantify the likelihood that the data can be described by a model with one-less class. } \\
\S \text { A putative role of concomitant variables only exists in models with at least two latent classes, in which concomitants can influence the } \\
\text { classification in different groups. } \\
\text { BIC, Bayesian information criteria; HF, heart failure; LCA, latent class analysis; Log L, log likelihood. }\end{array}$} \\
\hline
\end{tabular}

had low endorsement probabilities for all items (no symptoms and signs pattern; table 2).

The estimated proportion of subjects in classes 1, 2 and 3 defined without concomitant variables was $9.6 \%$, $19.2 \%$ and $71.1 \%$; when considering concomitant variables the estimated proportions were $10.1 \%, 27.8 \%$ and $62.1 \%$, respectively. When considering gender, age, education, obesity, diabetes and history of myocardial infarction or $\mathrm{HF}$, the discrimination to distinguish a third class increased mainly as a result of the reclassification of around a quarter of participants initially classified as non-cases into class 2, supporting the importance of including concomitant variables when judging the value of symptoms and signs of $\mathrm{HF}$.

Taking class 3 as reference, class 1 was positively associated with age $(\mathrm{OR}=1.07 /$ year $)$, obesity $(\mathrm{OR}=6)$, diabetes $(\mathrm{OR}=2.33)$ and history of myocardial infarction or HF $(\mathrm{OR}=12.94)$, and negatively associated with male sex $(\mathrm{OR}=0.11)$ and education ( $\mathrm{OR}=0.80 /$ year); class 2 was positively associated with age $(\mathrm{OR}=1.12 /$ year $)$ and obesity $(\mathrm{OR}=3.34)$. All these associations were statistically significant (table 2).

\section{Diagnostic value of clinical patterns}

The prevalence of left ventricular systolic dysfunction and left ventricular dilation was lower than $5 \%$, the prevalence of diastolic dysfunction, left ventricular hypertrophy, and left atrial dilation varied between $12 \%$ and $17 \%$, valvular disease affected $2.8 \%$ of the sample, and almost $30 \%$ had any of the former abnormalities on echocardiogram.

In general, the likelihood ratios showed that the patterns without concomitant variables changed pretest to post-test probability of cardiac abnormalities very little (minimum 0.6 for pattern 3 and maximum 4.1 for pattern 1). The area under the receiver operating characteristic (ROC) curve (AUC) for symptoms and signs patterns to predict the outcomes considered showed an overall improvement in discrimination of symptoms and signs patterns after the use of concomitant variables, largely at the expense of the likelihood ratio of pattern 3 , whose value for exclusion of $\mathrm{HF}$ increased (table 3). The negative likelihood ratios of pattern 3 were better than each individual symptoms and signs.

Pattern 1 is threefold more likely and pattern 3 is fivefold less likely in subjects with BNP above versus below $100 \mathrm{pg} / \mathrm{ml}$ (table 3), resulting in safe exclusion of high BNP in pattern 3 defined with concomitant variables.

\section{DISCUSSION}

In this study, the authors succeeded in identifying three patterns of syndromic aggregation of symptoms and signs for $\mathrm{HF}$, based on findings routinely collected in current clinical practice, by the application of LCA with concomitant variables to account for known determinants of the relevant clinical findings and the a priori probability of the condition. These models could be useful to standardise and quantify the probabilistic reasoning in clinical diagnosis, upon which decisions of further investigation and even treatment need to be made. Specifically, this model would be useful to rule out the need for requesting more accurate tools for assessment of cardiac function. In the case of epidemiological studies on HF, in which subjects are classified depending on a set of systematically collected data without the integrated view of one clinician to weigh the whole complex picture of a case, this model can be more useful to standardise the final classification of the subjects, and minimise the misclassification. These models allow weighting different combinations of symptoms and signs and, when using concomitant variables as we propose, taking into account variables that change the a priori probabilities, simulating the clinician reasoning. Additionally, there are usually a large number of observers in epidemiological studies and there is always some subjectivity of observer inherent to the classification. An additional major advantage of concomitant 
Table 2 Marginal percentage of subjects with each symptom and sign in each assigned latent class (pattern), with and without including concomitant variables (sex, age, education, obesity, diabetes and history of myocardial infarction or heart failure) to predict class membership, in the general population aged $\geq 45$ years, Porto, Portugal, $2006-$ 2008

\begin{tabular}{|c|c|c|c|c|c|c|c|}
\hline & \multirow[b]{2}{*}{ Total } & \multicolumn{3}{|c|}{ Pattern of symptoms and signs } & \multicolumn{3}{|c|}{$\begin{array}{l}\text { Pattern of symptoms and signs with } \\
\text { concomitant variables }\end{array}$} \\
\hline & & Class 1 & Class 2 & Class 3 & Class 1 & Class 2 & Class 3 \\
\hline & & 9.6 & 19.2 & 71.1 & 10.1 & 27.8 & 62.1 \\
\hline \multicolumn{8}{|l|}{ Dyspnoea } \\
\hline NYHA I & 86.9 & 18.3 & 91.4 & 95.5 & 20.9 & 90.6 & 96.9 \\
\hline NYHA II & 9.2 & 45.2 & 8.0 & 4.3 & 45.1 & 9.0 & 2.9 \\
\hline NYHA III & 3.9 & 36.4 & 0.7 & 0.2 & 33.9 & 0.4 & 0.2 \\
\hline \multicolumn{8}{|l|}{ Fatigue } \\
\hline No & 81.7 & 18.5 & 88.5 & 88.6 & 23.1 & 89.6 & 88.5 \\
\hline Yes & 18.3 & 81.5 & 11.5 & 11.4 & 76.9 & 10.4 & 11.5 \\
\hline \multicolumn{8}{|l|}{ Orthopnoea } \\
\hline No & 91.6 & 41.4 & 98.1 & 96.7 & 44.9 & 97.5 & 96.9 \\
\hline Yes, 1 pillow & 3.8 & 19.2 & 0.0 & 2.8 & 19.5 & 0.2 & 2.7 \\
\hline Yes, 2 or more & 4.7 & 39.4 & 1.9 & 0.5 & 35.6 & 2.2 & 0.4 \\
\hline \multicolumn{8}{|c|}{ pillows } \\
\hline \multicolumn{8}{|c|}{$\begin{array}{l}\text { Nocturnal paroxysmal } \\
\text { dyspnea }\end{array}$} \\
\hline No & 93.8 & 62.2 & 100.0 & 96.3 & 63.0 & 100 & 96.2 \\
\hline Yes & 6.2 & 37.8 & 0.0 & 3.7 & 37.0 & 0.0 & 3.8 \\
\hline \multicolumn{8}{|l|}{ Heart murmur } \\
\hline No & 93.9 & 87.6 & 91.1 & 95.8 & 86.1 & 90.6 & 97.0 \\
\hline Yes & 6.1 & 12.4 & 8.9 & 4.2 & 13.9 & 9.4 & 3.0 \\
\hline \multicolumn{8}{|l|}{ Pulmonary rales } \\
\hline No & 91.9 & 78.0 & 85.9 & 96.0 & 80.3 & 84.9 & 97.2 \\
\hline Yes & 8.1 & 22.0 & 14.1 & 4.0 & 19.7 & 15.1 & 2.8 \\
\hline \multicolumn{8}{|c|}{$\begin{array}{l}\text { Hepatojugular reflux or } \\
\text { jugular venous } \\
\text { distension }\end{array}$} \\
\hline No & 90.1 & 76.0 & 79.7 & 95.7 & 79.2 & 79.0 & 97.2 \\
\hline Yes & 9.9 & 24.0 & 20.3 & 4.3 & 20.8 & 21.0 & 2.8 \\
\hline \multicolumn{8}{|c|}{$\begin{array}{l}\text { Lower limb oedema at } \\
\text { the end of the day } \\
\text { (symptom) }\end{array}$} \\
\hline No & 73.2 & 35.3 & 55.5 & 84.6 & 35.0 & 61.0 & 85.5 \\
\hline Yes & 26.8 & 64.7 & 44.5 & 15.4 & 65.0 & 39.0 & 14.5 \\
\hline \multicolumn{8}{|c|}{$\begin{array}{l}\text { Lower limb oedema } \\
\text { (physical examination) }\end{array}$} \\
\hline No & 83.7 & 55.4 & 62.3 & 95.2 & 56.2 & 67.1 & 96.3 \\
\hline Ankle & 14.3 & 36.3 & 34.1 & 4.3 & 36.3 & 29.5 & 3.3 \\
\hline Up to the knee & 2.0 & 8.4 & 3.6 & 0.5 & 7.5 & 3.4 & 0.4 \\
\hline
\end{tabular}


Table 2 Continued

\begin{tabular}{|c|c|c|c|c|c|c|c|}
\hline & \multirow[b]{2}{*}{ Total } & \multicolumn{3}{|c|}{ Pattern of symptoms and signs } & \multicolumn{3}{|c|}{$\begin{array}{l}\text { Pattern of symptoms and signs with } \\
\text { concomitant variables }\end{array}$} \\
\hline & & Class 1 & Class 2 & Class 3 & Class 1 & Class 2 & Class 3 \\
\hline \multicolumn{8}{|l|}{$\begin{array}{l}\text { Trophic signs of chronic } \\
\text { venous insufficiency }\end{array}$} \\
\hline No & 83.8 & 67.7 & 50.2 & 97.6 & 68.0 & 60.4 & 97.3 \\
\hline Yes & 16.2 & 32.3 & 49.8 & 2.4 & 32.0 & 39.6 & 2.7 \\
\hline \multicolumn{8}{|l|}{ Visible varicose veins } \\
\hline No & 58.5 & 44.3 & 15.1 & 75.5 & 40.2 & 23.5 & 77.9 \\
\hline Yes & 41.5 & 55.7 & 84.9 & 24.5 & 59.8 & 76.5 & 22.1 \\
\hline \multicolumn{5}{|l|}{ Concomitant variables } & \multicolumn{3}{|c|}{ Multinomial logistic regression } \\
\hline & & & & & OR $(95 \% \mathrm{Cl})$ & OR $(95 \%$ & \\
\hline Sex (male) & & & & & 0.11 (0.04 to 0.32$)$ & $0.54(0.2$ & $\operatorname{Ref}^{*}$ \\
\hline Age (per year) & & & & & 1.07 (1.03 to 1.12$)$ & $1.12(1.0$ & $\operatorname{Ref}^{*}$ \\
\hline Education (per year) & & & & & $0.80(0.70$ to 0.91$)$ & $0.99(0.9$ & $\operatorname{Ref}^{*}$ \\
\hline Obesity & & & & & $6.00(2.85$ to 12.64$)$ & $3.34(1.4$ & $\operatorname{Ref}^{*}$ \\
\hline Diabetes & & & & & 2.33 (1.07 to 5.08$)$ & $0.61(0.2$ & $\operatorname{Ref}^{*}$ \\
\hline $\begin{array}{l}\text { History of myocardial } \\
\text { infarction or heart } \\
\text { failure }\end{array}$ & & & & & 12.94 (4.95 to 33.80$)$ & 1.60 & $\operatorname{Ref}^{*}$ \\
\hline
\end{tabular}


Table 3 Likelihood ratio and predictive value (\%) of patterns of symptoms and signs with and without concomitant variables, for the presence of objective cardiac structural and functional parameters. Area under the ROC curve for the classification with and without concomitants

\begin{tabular}{|c|c|c|c|c|c|c|c|c|c|}
\hline & \multirow{2}{*}{$\begin{array}{l}\text { Outcomes } \\
\text { prevalence } \\
\text { N (\%) }\end{array}$} & \multicolumn{3}{|c|}{ Pattern of symptoms and signs } & \multirow[b]{2}{*}{ AUC } & \multicolumn{3}{|c|}{$\begin{array}{l}\text { Pattern of symptoms and signs } \\
\text { with concomitant variables }\end{array}$} & \multirow[b]{2}{*}{ AUC } \\
\hline & & $\begin{array}{l}1 \\
\text { LR (PV) }\end{array}$ & $\begin{array}{l}2 \\
\text { LR (PV) }\end{array}$ & $\begin{array}{l}3 \\
\text { LR (PV) }\end{array}$ & & $\begin{array}{l}1 \\
\text { LR (PV) }\end{array}$ & $\begin{array}{l}2 \\
\text { LR (PV) }\end{array}$ & $\begin{array}{l}3 \\
\text { LR (PV) }\end{array}$ & \\
\hline \multicolumn{10}{|l|}{ Outcomes } \\
\hline $\begin{array}{l}\text { Left ventricular systolic } \\
\text { dysfunction }\end{array}$ & $39(3.5)$ & $3.6(11.5)$ & $1.1(3.9)$ & $0.6(2.3)$ & 64.5 & $3.4(11.1)$ & $1.1(3.7)$ & $0.6(2.2)$ & 65.1 \\
\hline Left ventricular dilation & $55(4.9)$ & $4.1(16.8)$ & $1.2(5.6)$ & $0.6(2.8)$ & 67.4 & $4.4(17.7)$ & $1.0(4.9)$ & $0.5(2.5)$ & 69.5 \\
\hline Diastolic dysfunction & $161(14.5)$ & $3.6(38.0)$ & $1.6(21.9)$ & $0.6(9.8)$ & 65.2 & $3.6(38.1)$ & $1.7(22.5)$ & $0.5(7.6)$ & 69.3 \\
\hline $\begin{array}{l}\text { Left ventricular } \\
\text { hypertrophy }\end{array}$ & $145(12.9)$ & $3.4(33.6)$ & $1.3(16.4)$ & $0.7(9.4)$ & 63.0 & $3.5(34.5)$ & $1.4(17.8)$ & $0.5(7.4)$ & 67.5 \\
\hline Left atrium dilation & $185(16.6)$ & $2.2(30.8)$ & $1.5(23.8)$ & $0.7(13.1)$ & 60.0 & $2.4(33.0)$ & $1.5(23.0)$ & $0.6(11.5)$ & 62.9 \\
\hline Valvular disease & $31(2.8)$ & $4.0(10.6)$ & $0.7(1.9)$ & $0.7(2.1)$ & 63.0 & $3.4(9.0)$ & $1.5(4.3)$ & $0.4(1.2)$ & 71.1 \\
\hline Any of the above & $322(29.6)$ & $2.6(52.4)$ & $1.5(38.5)$ & $0.8(24.2)$ & 60.0 & $3.1(56.9)$ & $1.6(39.9)$ & $0.6(20.7)$ & 64.5 \\
\hline High $\operatorname{BNP}(\geq 100 \mathrm{pg} / \mathrm{ml})$ & $40(6.3)$ & $3.6(19.4)$ & $1.8(10.7)$ & $0.4(2.6)$ & 72.9 & $3.4(18.2)$ & $1.7(9.9)$ & $0.2(1.7)$ & 75.1 \\
\hline
\end{tabular}

variable LCA models is that this integration of individual items into a classification is standardised, maximising the reliability and expectedly minimising the misclassification.

In general, the likelihood ratios showed that the symptoms and signs patterns generated only relatively small changes from pretest to post-test probability of cardiac abnormalities. This was expected, considering that many of these abnormalities are known to be asymptomatic in a large proportion of patients for a long time and the symptoms are unspecific, and is compatible with previous quantifications of the value of symptoms and signs. ${ }^{27}$ The patterns showed a small diagnostic value and a high value to exclude a high $\mathrm{BNP}$ value, an established biomarker for the diagnosis of HF.

The LCA is a probabilistic approach to disease classification which allows the identification of more precise categories of disease conditions. ${ }^{28}$ This framework brings several statistical advantages over standard classification approaches not supervised (cluster analysis). First, it allows problems such as the choice of the number of classes and of the classification method to be recast as statistical model choice problems. Second, LCM can be potentially improved through the use of concomitant variables, that is, variables that influence the prevalence of classes, thus permitting the identification of more precise categories. Finally, for given values of the response and concomitant variables, posterior class membership probabilities for each individual are produced. It has been used to validate diagnostic tests in the absence of a perfect reference standard, ${ }^{28}$ which is the situation for HF. A new feature of this study is that for the first time, to our knowledge, these classifications integrated factors which have a large impact on the prevalence of symptoms and signs suggestive of HF. The novelty in our application is that class probabilities are adjusted for concomitant variables. Specifically, the model estimates the increase or decrease in class probabilities for individuals conditional to the respective concomitant variables pattern, contributing to increased discrimination and a decrease in the number of falsenegatives and false-positives. The inclusion of these variables improved model fit. The area under the ROC curve (AUC) for symptoms and signs patterns to predict the outcomes considered showed an overall improvement in discrimination of symptoms and signs patterns after the use of concomitant variables, largely at the expense of the likelihood ratio of pattern 3 (no symptoms and signs) to exclude cardiac abnormalities. The inclusion of concomitant variables helped to refine class 3 (reclassifying individuals from class 3 to class 2, thus decreasing the false-negatives). These results objectively indicate that the use concomitant variables can improve the diagnostic value of the symptoms and signs patterns and, consequently, improve the usefulness of the symptoms and signs for diagnosis and as an outcome measures. The potential for application in other settings of complex diagnoses is very high.

In patterns with concomitant variables, class 1 was more prevalent in women, individuals with a history of myocardial infarction or $\mathrm{HF}$, diabetic and obese individuals, increased with age and decreased with education, while class 2 only increased with age and obesity. These associations reflect the influence of gender and education on subjective importance attributed to symptoms, ${ }^{15}$ likely a psychosocial effect, while age, obesity, diabetes and history of myocardial infarction or HF are biologically associated with decreasing cardiac function even at asymptomatic stages.

The patterns identified by this methodological approach depend on the type of population being studied. In this study, in a sample of the general 
population, three patterns were identified: symptomatic HF pattern, symptoms and signs of congestion and no symptoms and signs. In a previous study, using a similar approach in subjects discharged after myocardial infarction or acute HF, the authors were able to distinguish different patterns (non-cases, HF and advanced HF). ${ }^{12}$ The patterns identified in the current study are appropriate for diagnosis in the general population only. The low prevalence of advanced and severe $\mathrm{HF}$ cases is a limitation of this study and could have underestimated the discriminative capacity of this set of items. Also, the prevalence of more specific symptoms and signs such as a third heart sound ${ }^{29}$ was too low in this sample to be able to take them into account, suggesting that the proposed patterns are likely to be more sensitive but less specific than previously available scores such as the Framingham criteria, supporting their usefulness as potential screening tools or initial clinical investigation that do not aim to replace full investigation in the clinical setting. A valid assessment of diastolic dysfunction, currently recommended for the diagnosis of $\mathrm{HF}^{18}$ using up-to-date technology, is a major advantage of this study. The prevalence of hypertension is very large in this population, ${ }^{30}$ while this is a low coronary heart disease risk country, expectedly increasing the burden of diastolic abnormalities. Since mild diastolic changes have more questionable clinical significance, ${ }^{31}$ though discrimination of such mild cases would require technological means that were unavailable in our study such as study of pulmonary veins flow, ${ }^{18}$ this could have contributed to a less favourable performance of the clinical diagnosis in this setting.

The role of BNP testing is clearly defined for diagnosing patients with suspected $\mathrm{HF}^{32}$ Ventricular wall stretch is the major determinant of increased BNP concentrations and peptide levels have limited accuracy in differentiating HF with left ventricular systolic dysfunction or with preserved ejection fraction. ${ }^{33}$ The high sensitivity of the model with concomitant variables is supported by the virtually null prevalence of high BNP among subjects classified as no symptoms and signs pattern. However, a large proportion of participants classified in classes 1 and 2 still did not have BNP above $100 \mathrm{pg} / \mathrm{ml}$, arguing in favour of the likely existence of false positives in these patterns.

The fact that validation by comparison with echocardiographic parameters and BNP values was performed using the same sample in which the LCA models were fit is a limitation of this study. However, all clinical and echocardiographic data were collected blinded to each other and to BNP values, preventing an artificially high correlation between the LCA and both cardiac abnormalities and the biomarker, due to the subjective and observer-dependent nature of the items being assessed.

Future developments of this research work aim at translating the validated patterns into a classification score, using an approach for making complex statistical models useful to practitioners and researchers, such as a circular ruler $^{34}$ or points system, ${ }^{35}$ which was used for example to develop the widely used Framingham risk scores. Use of this tool will allow the identification of high-risk candidates for HF who are likely to have a substantial yield of positive findings when tested for objective measures of cardiac dysfunction in clinical practice, as well as to confidently exclude $\mathrm{HF}$ in others, thus orienting the clinical investigation in alternative directions. Such a tool could also increase discrimination and decrease the number of false-negatives and false-positives in epidemiological studies on HF, in which subjects are classified depending on a set of systematically collected data without the integrated view of one clinician to weigh the whole complex picture of a case.

\section{Author affiliations}

${ }^{1}$ Department of Clinical Epidemiology, Predictive Medicine and Public Health, University of Porto Medical School, Porto, Portugal

${ }^{2}$ Institute of Public Health of the University of Porto, Porto, Portugal

${ }^{3}$ Department of Mathematics, University of Porto Science School, Porto,

Portugal

${ }^{4}$ Mathematics Center, University of Porto, Porto, Portugal

${ }^{5}$ Department of Internal Medicine, Heart Failure Clinic, Hospital São João,

Porto, Portugal

${ }^{6}$ Department of Cardiology, Hospital São João, Porto, Portugal

Contributor MS analysed and interpreted the data, and wrote the first draft of the article. $R G$ analysed and interpreted the data and reviewed the article. PL, MA and AG substantially contributed to acquisition of data and reviewed the article. NL substantially contributed to interpretation of the data and revised the article critically for important intellectual content. PB conceived and designed the study, collected data and revised the article for important intellectual content. AA conceived and designed the study, analysed and interpreted the data and drafted the article. All authors gave final approval of the version to be published.

Competing interests None.

Ethics approval The local ethics committee approved the study, Hospital S. João.

Funding Two grants, from Fundação Astra Zeneca and Fundação para a Ciência e a Tecnologia (POCI/SAU-ESP/61492/2004), are gratefully ackowledged.

Provenance and peer review Not commissioned; externally peer reviewed.

Data sharing statement No additional data are available.

\section{REFERENCES}

1. Dickstein K, Cohen-Solal A, Filippatos G, et al. ESC Guidelines for the diagnosis and treatment of acute and chronic heart failure 2008: the Task Force for the Diagnosis and Treatment of Acute and Chronic Heart Failure 2008 of the European Society of Cardiology. Developed in collaboration with the Heart Failure Association of the ESC (HFA) and endorsed by the European Society of Intensive Care Medicine (ESICM). Eur Heart J 2008;29:2388-442.

2. Hunt SA. ACC/AHA 2005 guideline update for the diagnosis and management of chronic heart failure in the adult: a report of the American College of Cardiology/American Heart Association Task Force on Practice Guidelines (Writing Committee to Update the 2001 Guidelines for the Evaluation and Management of Heart Failure). J Am Coll Cardiol 2005;46:e1-82.

3. Hobbs FD, Doust $\mathrm{J}$, Mant $\mathrm{J}$, et al. Heart failure: diagnosis of heart failure in primary care. Heart ;96:1773-7.

4. Mant $\mathrm{J}$, Doust $\mathrm{J}$, Roalfe A, et al. Systematic review and individual patient data meta-analysis of diagnosis of heart failure, with modelling of implications of different diagnostic strategies in primary care. Health Technol Assess 2009;13:1-207, iii. 
5. Mosterd A, Deckers JW, Hoes AW, et al. Classification of heart failure in population based research: an assessment of six heart failure scores. Eur J Epidemiol 1997;13:491-502.

6. McKee PA, Castelli WP, McNamara PM, et al. The natural history of congestive heart failure: the Framingham study. N Engl J Med 1971;285:1441-6.

7. Eriksson $\mathrm{H}$, Caidahl K, Larsson B, et al. Cardiac and pulmonary causes of dyspnoea-validation of a scoring test for clinical-epidemiological use: the Study of Men Born in 1913. Eur Heart J 1987;8:1007-14

8. Walma EP, Hoes AW, Prins A, et al. Withdrawing long-term diuretic therapy in the elderly: a study in general practice in The Netherlands. Fam Med 1993;25:661-4.

9. Schocken DD, Arrieta MI, Leaverton PE, et al. Prevalence and mortality rate of congestive heart failure in the United States. J Am Coll Cardiol 1992;20:301-6.

10. Gheorghiade M, Beller GA. Effects of discontinuing maintenance digoxin therapy in patients with ischemic heart disease and congestive heart failure in sinus rhythm. Am J Cardiol 1983;51:1243-50.

11. Carlson KJ, Lee DC, Goroll AH, et al. An analysis of physicians reasons for prescribing long-term digitalis therapy in outpatients. $J$ Chronic Dis 1985;38:733-9.

12. Kim J, Jacobs DR Jr, Luepker RV, et al. Prognostic value of a novel classification scheme for heart failure: the Minnesota Heart Failure Criteria. Am J Epidemiol 2006;164:184-93.

13. Remes $\mathrm{J}$, Miettinen $\mathrm{H}$, Reunanen $\mathrm{A}$, et al. Validity of clinical diagnosis of heart failure in primary health care. Eur Heart $J$ 1991;12:315-21.

14. Wheeldon NM, MacDonald TM, Flucker CJ, et al. Echocardiography in chronic heart failure in the community. QJM 1993;86:17.

15. Azevedo A, Bettencourt P, Pimenta J, et al. Clinical syndrome suggestive of heart failure is frequently attributable to non-cardiac disorders_-population-based study. Eur J Heart Fail 2007;9:391-6.

16. Bianchi MT, Alexander BM. Evidence based diagnosis: does the language reflect the theory? BMJ 2006;333:442-5.

17. Petrie M, McMurray J. Changes in notions about heart failure. Lancet 2001;358:432-4.

18. Paulus WJ, Tschope C, Sanderson JE, et al. How to diagnose diastolic heart failure: a consensus statement on the diagnosis of heart failure with normal left ventricular ejection fraction by the Heart Failure and Echocardiography Associations of the European Society of Cardiology. Eur Heart J 2007;28:2539-50.

19. Owan TE, Hodge DO, Herges RM, et al. Trends in prevalence and outcome of heart failure with preserved ejection fraction. $N$ Engl $J$ Med 2006;355:251-9.

20. Ramos E, Lopes C, Barros H. Investigating the effect of nonparticipation using a population-based case-control study on myocardial infarction. Ann Epidemiol 2004;14:437-41.
21. Severo M, Gaio R, Lourenco P, et al. Indirect calibration between clinical observers-application to the New York Heart Association functional classification system. BMC Res Notes 2011;4:276.

22. Clinical guidelines on the identification, evaluation, and treatment of overweight and obesity in adults: executive summary. Expert panel on the identification, evaluation, and treatment of overweight in adults. Am J Clin Nutr 1998;68:899-917.

23. Severo M, Pereira M, Bettencourt P, et al. B-type natriuretic peptide measured in serum - calibration using plasma samples for research purposes. Clin Lab 2011;57:1015-19.

24. Zaphiriou A, Robb S, Murray-Thomas T, et al. The diagnostic accuracy of plasma BNP and NTproBNP in patients referred from primary care with suspected heart failure: results of the UK natriuretic peptide study. Eur J Heart Fail 2005;7:537-41.

25. Kuch B, Schunkert $H$, Muscholl $M$, et al. Distribution, determinants and reference values of left ventricular parameters in the general population-results of the MONICA/KORA echocardiography studies. Gesundheitswesen 2005;67(Suppl 1):S68-73.

26. Vermunt JK, Magidson J. Latent class cluster analysis. Appl Latent Class Anal 2002:89-106

27. Wang CS, FitzGerald JM, Schulzer M, et al. Does this dyspneic patient in the emergency department have congestive heart failure? JAMA 2005;294:1944-56.

28. Guggenmoos-Holzmann I, van Houwelingen HC. The (in)validity of sensitivity and specificity. Stat Med 2000;19:1783-92.

29. Wang CS, FitzGerald JM, Schulzer M, et al. Does this dyspneic patient in the emergency department have congestive heart failure? JAMA 2005;294:1944.

30. Pereira $\mathrm{M}$, Carreira $\mathrm{H}$, Vales $\mathrm{C}$, et al. Trends in hypertension prevalence (1990-2005) and mean blood pressure (1975-2005) in Portugal: a systematic review. Blood Press 2012;21:220-6.

31. Goncalves A, Almeida PB, Lourenco P, et al. Clinical significance of impaired relaxation pattern in middle-aged and elderly adults in the general population. Rev Port Cardiol 2010;29:1799-806.

32. Bettencourt PM. Clinical usefulness of B-type natriuretic peptide measurement: present and future perspectives. Heart 2005;91:1489-94.

33. Maisel AS, McCord J, Nowak RM, et al. Bedside B-Type natriuretic peptide in the emergency diagnosis of heart failure with reduced or preserved ejection fraction. Results from the Breathing Not Properly Multinational Study. J Am Coll Cardiol 2003;41:2010-17.

34. Severo M, Lopes C, Lucas R, et al. Development of a tool for the assessment of calcium and vitamin $\mathrm{D}$ intakes in clinical settings. Osteoporos Int 2009;20:231-7.

35. Sullivan LM, Massaro JM, D'Agostino RB Sr. Presentation of multivariate data for clinical use: the Framingham Study risk score functions. Stat Med 2004;23:1631-60. 